\title{
Immunohistochemical Analysis of Citrulline-Nitric Oxide Cycle Enzymes and Glutamine Synthetase in Different Regions of Brain in Epilepsy Rat Model
}

\author{
Mummedy Swamy*, Intan Nurfirdaus Mat Zin, K. N. S. Sirajudeen, Zulkarnain Mustapha, \\ Chandran Govindasamy \\ Department of Chemical Pathology, School of Medical Sciences, Health Campus, Universiti Sains Malaysia, \\ Kelantan, Malaysia \\ Email: ․ㅗswamy@usm.my, mummedys@yahoo.co.in
}

Received 17 April 2014; revised 10 May 2014; accepted 5 June 2014

Copyright (C) 2014 by authors and Scientific Research Publishing Inc. This work is licensed under the Creative Commons Attribution International License (CC BY). http://creativecommons.org/licenses/by/4.0/

(c) (i) Open Access

\section{Abstract}

The aim of this study was to determine the immunoreactivity of neuronal and inducible nitric oxide synthetase, argininosuccinate synthetase, argininosuccinate lyase, glutamine synthetase in different regions of brain in rats of kainic acid mediated epilepsy. Male Sprague-Dawley rats were used in this study. The acute group animals were sacrificed after 2 hours and the chronic group animals were sacrificed after 5 days of a single subcutaneous injection of kainic acid $(15 \mathrm{mg} / \mathrm{kg}$ body weight). The cerebral cortex, cerebellum and brain stem slices were fixed and immunohistostained for the above enzymes. Images were captured and analyzed. In acute group, argininosuccinate synthetase and inducible nitric oxide synthetase were increased in cerebral cortex and cerebellum, neuronal nitric oxide synthetase increased in cerebral cortex and brain stem, and there was no change in argininosuccinate lyase immunoreactivity compared to control group. In chronic group, glutamine synthetase was decreased and all other enzymes immunoreactivity was increased in all the brain regions tested. This study demonstrated the up-regulation of citrulline-nitric oxide cycle enzymes and may contribute to enhancing recycling of citrulline to arginine to support the increased production of nitric oxide in epilepsy. The decreased glutamine synthetase may increase glutamate in chronic epilepsy and may lead to neurodegeneration.

\section{Keywords}

Citrulline-Nitric Oxide Cycle Enzymes, Epilepsy, Glutamine Synthetase, Immunohistochemistry,

"Corresponding author. 


\section{Kainic Acid, Rat Brain}

\section{Introduction}

Nitric oxide (NO) is postulated to be involved in the pathophysiology of different epilepsy models. NO is synthesized from L-arginine by three isoforms of nitric oxide synthase (NOS) and the citrulline formed as a co-product may be used for arginine production by the action of argininosuccinate synthetase (AS) and argininosuccinate lyase (AL) through citrulline-NO cycle [1]. The induction of AS, cationic amino acid transporter-2, and NOS in activated murine microglial cells [2] and induction of inducible NOS, AS and AL in cytokine-stimulated PC12 cells and high production of NO were earlier reported by Zhang et al. [1]. Kainic acid (KA) is a powerful excitotoxin, and produces acute and sub-acute epileptic activity, ultimately resulting in widespread irreversible neuropathological changes [3]. The mechanisms contributing to high concentration of NO in epilepsy are not well understood. It was shown that NOS knockout mice were more severely affected by epileptic activity than controls and the response to NO during epilepsy depends on its concentration and NO may be regarded as an anticonvulsant and proconvulsant substance in relation to convulsions induced by pentylenetetrazole (PTZ) [4]. In the CNS increased excitatory glutamate receptors, activation is considered as an important mechanism in neurodegenerative disorders and the conversion of glutamate to glutamine by glutamine synthetase (GS), that takes place within the astrocytes, represents a key mechanism in the regulation of excitatory neurotransmission under physiological as well as pathological conditions in brain [5]. Earlier studies reported the nitration and inhibition of GS during PTZ induced seizure model at repeated PTZ seizure induction, but there was no change in protein concentration [6].

However, there were gene expression studies indicating decreased GS expression in chronic phase of epilepsy induced by KA [7]. It is also reported that haploinsufficiency of GS increases susceptibility to experimental febrile seizures [8]. It is earlier reported the decreased activity of GS and increased activities of NOS, AS, AL in acute and chronic groups of KA mediated epilepsy [9]. Therefore the present study was conducted to analyze immunohistochemically the expression of nNOS, iNOS, AS, AL and GS in cerebral cortex (CC), cerebellum (CB) and brain stem (BS) of rats in acute and chronic groups of KA mediated epilepsy.

\section{Material and Methods}

\subsection{Animals and Epilepsy Induction}

Male Sprague Dawley rats weighing 200 - 250 grams were used for the study and these were obtained from the animal research and service center, Health campus, Universiti Sains Malaysia, Malaysia. The animals had free access to food and water. Animal ethics committee of Universiti Sains Malaysia, Health campus, Kubang Kerian, Malaysia, approved the experimental design and number of animals [USM/Animal Ethics Approval/2007/(34) (105)]. The animals were divided into control, acute group and chronic groups ( $n=6$ rats/group). In the acute group, epilepsy was produced by subcutaneous administration of KA $(15 \mathrm{mg} / \mathrm{kg}$ body weight, dissolved in normal saline). KA was obtained from Sigma Chemical Company, USA. Control group received normal saline subcutaneously [10]. The animals showed convulsions after 40 - 45 min of KA injection for 2 - 3 min and afterwards became drowsy. The animals were sacrificed after 2 hours of injection using the guillotine. In the chronic group, the animals were given a KA subcutaneous injection (single dose of $15 \mathrm{mg} / \mathrm{kg}$ body weight, dissolved in normal saline) on day one and they were given free access to food and water during the next 5 days before sacrifice. After sacrificed the brains were quickly removed, placed in ice cold saline and blotted with filter paper to remove blood and the different regions (CC, CB and BS) were separated as described by Sadasivudu and Lajtha [11].

\subsection{Immunohistochemistry Staining for NOS (nNOS and iNOS), AL, AS and Glutamine Synthethase}

The brain tissues were constructed using formalin-fixed, paraffin embedded and then sectioned at $4 \mu \mathrm{m}$, transferred onto poly-L-lysine slides. Immunohistochemical staining was done according to the standard AvidinBiotin Complex (ABC) staining technique [12]. Slides of samples were washed in xylene to remove paraffin, 
rehydrated in serial dilutions of alcohols and washed with distilled water. Xylene was obtained from Merck, Germany. All the other reagents used were analytical grade by the local chemical suppliers. Samples soaked in 3\% hydrogen peroxide to prevent endogenous activity. Antigen retrieved by putting the samples into sodium citrate buffer (pH 6.0) and heated in microwave for 2 times 10 minutes sessions. Samples were then incubated with polyclonal primary antibody (Santa Cruz, CA, USA) overnight at $4^{\circ} \mathrm{C}$ and continued with secondary antibody for 1 hour. The Streptavidin peroxidise method (rabbit ABC Staining System: sc-2018, Santa Cruz, CA, USA) performed for signal development and samples counterstained with hematoxylin. Positive control was gained from brown stained cells of brain tissues and negative control done by excluding the primary antibody. Slides were mounted with DPX mounting media for observation and captured by Olympus XC50 (Shinjuku, Tokyo, Japan).

\subsection{Immunohistochemistry Analysis}

Positive stained cells were counted using (analysis Software 5.0) in an area approximately $0.1 \mathrm{~cm}^{2}$. By using the software, a rectangle box was drawn manually on the picture (magnification of $10 \times$ ) and the total numbers of positive cells were counted. Two slides per brain region and three areas per slide (i.e. $3 \times 2=6$ observation per animal) and 36 observations per group were averaged [13].

\subsection{Statistical Analysis}

Results were reported as mean \pm standard error of mean (SEM) values from 6 animals for each parameter calculated for each group. Statistical analysis was carried out by using one-way analysis of variance (ANOVA) followed by Bonferroni post hoc test, using the SPSS software (version 12.0.1) $\mathrm{p}$ value of $\mathrm{p}<0.05$ was considered as statistically significant at $95 \%$ confidence interval.

\section{Results}

The immunoreactivity of AS, AL, nNOS, iNOS and GS in different regions of rat brain in control, acute and chronic groups of KA mediated epilepsy were given in Figures 1-5 respectively and their immunoreactivity analysis was given in Figure 6. In acute group, AS and iNOS immunoreactivity (Figure 1, Figure 4 and Figure 6) were increased in CC and CB, nNOS immunoreactivity (Figure 3 and Figure 6) increased in CC and BS, and there was no change in AL immunoreactivity (Figure 2 and Figure 6) compared to control group. In chronic group AS, AL, nNOS and iNOS immunoreactivity was increased when compared to control in all the brain regions tested (Figures 1-6). GS immunoreactivity (Figure 5 and Figure 6) was decreased in all the three brain regions in chronic group and $\mathrm{CB}$ in acute group.

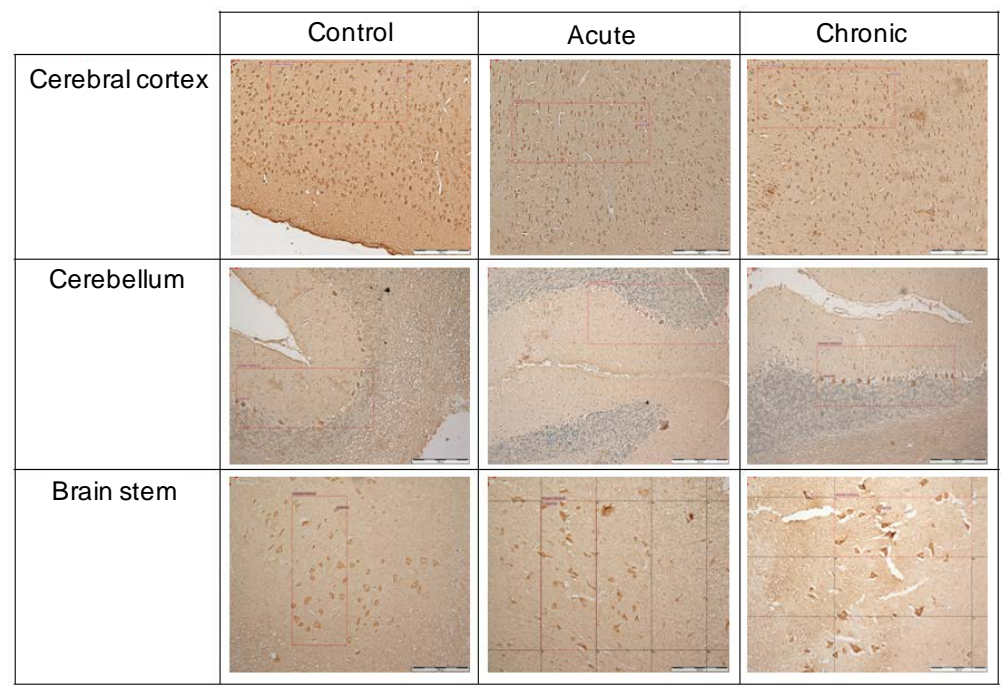

Figure 1. Immunoreactivity AS in different regions of rat brain in control, acute and chronic groups of KA mediated epilepsy. 


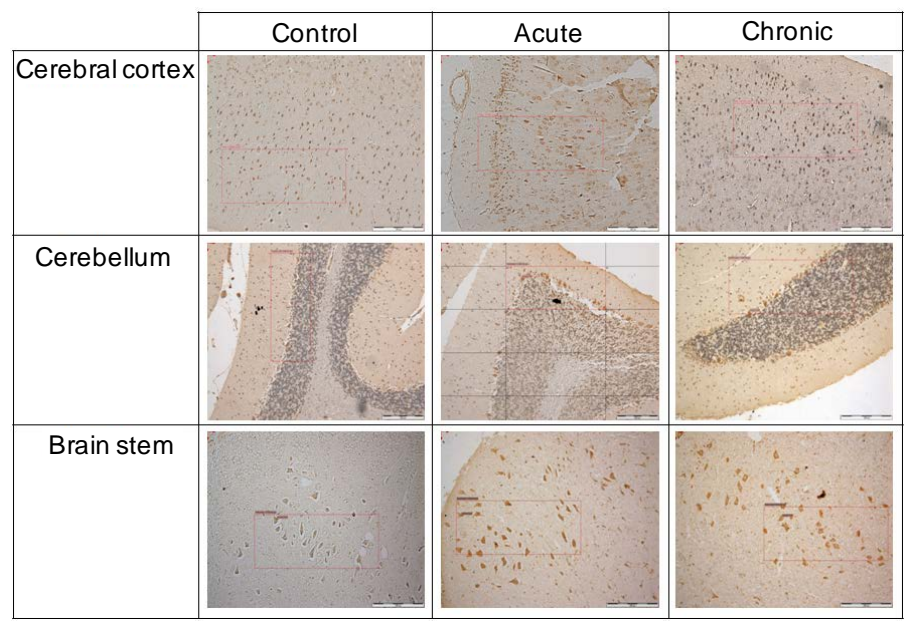

Figure 2. Immunoreactivity of AL in different regions of rat brain in control, acute and chronic groups of KA mediated epilepsy.

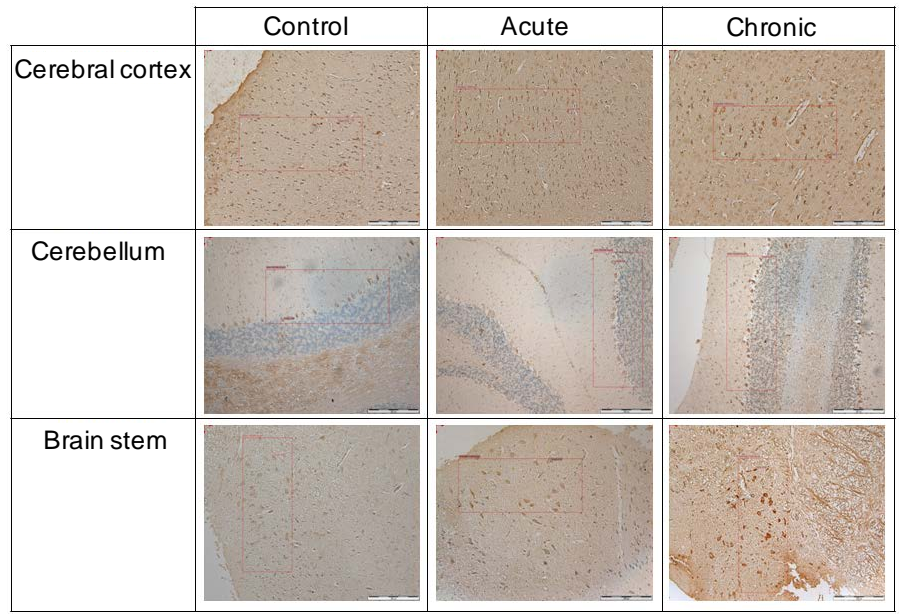

Figure 3. Immunoreactivity of nNOS in different regions of rat brain in control, acute and chronic groups of KA mediated epilepsy.

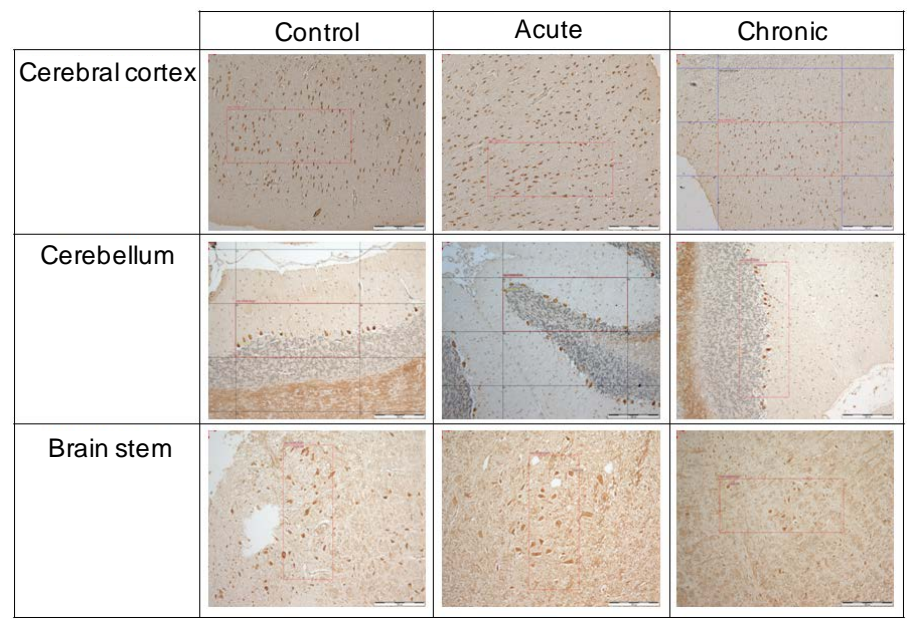

Figure 4. Immunoreactivity of iNOS in different regions of rat brain in control, acute and chronic groups of KA mediated epilepsy. 


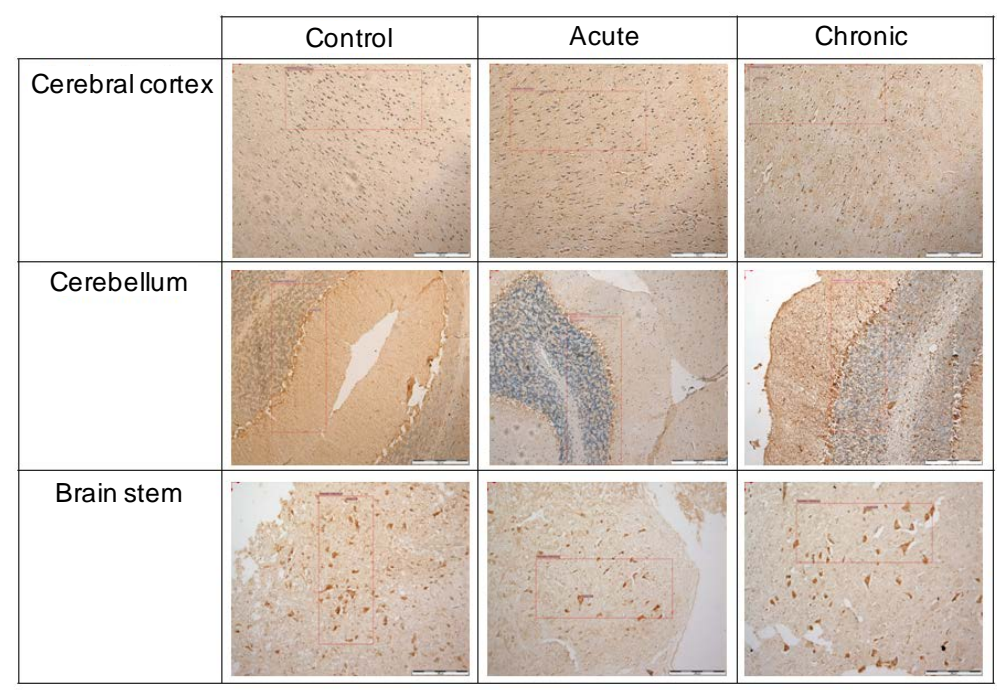

Figure 5. Immunoreactivity of GS in different regions of rat brain in control, acute and chronic groups of KA mediated epilepsy.

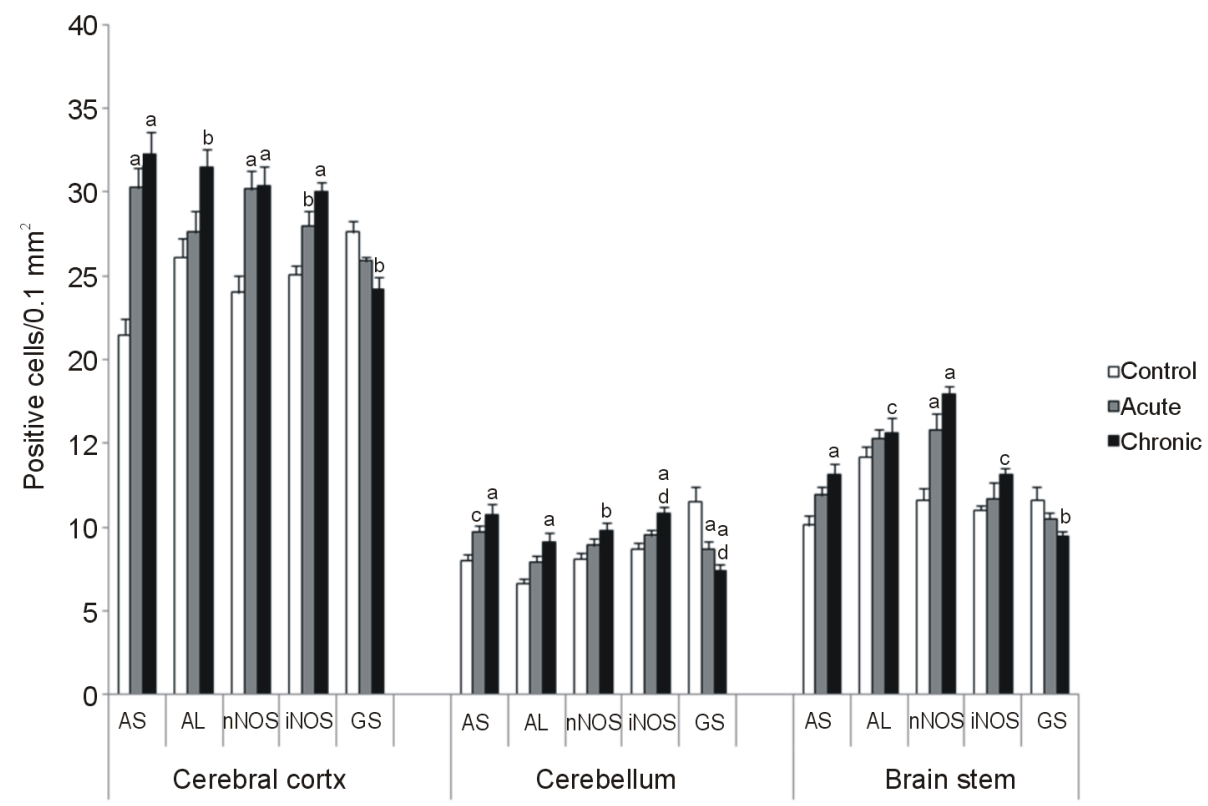

Figure 6. Immmunoreactivity analysis AS, AL, nNOS, iNOS and GS in different regions of rat brain in control, acute and chronic groups of KA mediated epilepsy. Statistical analysis was carried out by using one-way analysis of variance (ANOVA) followed by Bonferroni post hoc test; Values are mean \pm standard error of mean (SEM) from 6 animals in each group. ${ }^{\mathrm{a}} \mathrm{p}<$ $0.001,{ }^{b} \mathrm{p}<0.01$ and ${ }^{\mathrm{c}} \mathrm{p}<0.05$ versus control group; ${ }^{\mathrm{d}} \mathrm{p}<0.05$ versus acute group.

\section{Discussion}

Neuronal NO generation is implicated in the pathogenesis of both direct and secondary excitotoxic neuronal injuries in vivo. NMDA receptors may be contributing critically to neuronal injury in various acute conditions, but several studies support the hypothesis that AMPA/KA receptors may be of greater importance to the neurodegenerative process [14]. Earlier studies reported a high production of NO in cortex than in amygdala and hippocampus, eventhogh there are most kainate receptors in the hippocampus [10]. The increased levels of NO in brain in epilepsy indicate the involvement of NO in pathophysiology of excitotoxicity [9] [10] [15] [16]. The increased production of NO in acute as well as in chronic group indicates the continuous increased production of 
NO and its deleterious effects in CNS in chronic conditions of epilepsy [9]. The increased iNOS immunoreactivity observed in all brain regions in chronic group in this study supports the increased activity of NOS and increased iNOS mRNA expression reported [9] [17]. The increased iNOS immunoreactivity along with AS and $\mathrm{AL}$ immunoreactivity in chronic group of this study indicates the co-expression of these enzymes in epilepsy. Such a co-induction of iNOS, AS and AL were reported earlier in cytokine-stimulated PC12 cells [1].

In the CNS increased excitatory glutamate receptors, activation is considered as an important mechanism in neurodegenerative disorders [18] [19]. The conversion of glutamate to glutamine by GS, that takes place within the astrocytes, represents a key mechanism in the regulation of excitatory neurotransmission under physiological as well as pathological conditions in brain [5]. The GS is present in all regions of brain and it is high in cerebral cortex, cerebellum and hippocampus [20] [21]. The modulation of GS activity is important and its impairment or saturation can cause pathological consequences in brain [22]. The GS activity was decreased in acute and chronic groups of epilepsy [9]. The mechanisms of inhibition of GS by NO are not clear, but it is reported to be as a covalent modification due to nitrosylation or nitration of tyrosine in GS [21] [23]. Earlier studies reported the nitration and inhibition of GS during PTZ induced seizure model at repeated PTZ seizure induction, but there was no change in protein concentration [6], however, there were gene expression studies indicating decreased GS expression in chronic phase of epilepsy induced by KA [7] [17]. The decreased GS immunoreactivty observed in all the brain regions of chronic group in this study support the earlier report of decreased GS expression [7] [17]. The results of GS immunoreactivty in acute group did not show any change in CC and BS, hence the reported decrease of GS activity [15] may be due to its nitration by NO in this condition. It is also reported that haploinsufficiency of GS increases susceptibility to experimental febrile seizures [8]. The decreased GS immunoreactivty observed in this study and decreased activity and mRNA expression reported earlier may contribute for prolonged availability of glutamate for excitotoxicity [9] [16] [17]. The increased formation of NO along with increased activities and mRNA expression of NOS, AS, and AL reported earlier [9] [16] [17] and the immunoreactivty results presented in this study are in agreement with increased formation of NO in KA mediated epilepsy and NO involvement in modulation of GS. It is indicated that the decreased GS may provide sustained availability of glutamate, which may contribute to excitotoxicity in chronic epilepsy.

\section{Conclusion}

The study clearly shown the increased immunoreactivty of nNOS and iNOS in all the brain regions studied and indicated a complimentary condition for increased NO synthesis in chronic epilepsy. The increased immunoreactivty of AS and AL in all the three brain regions studied in chronic group may contribute for increased recycling of citrulline to arginine which may support the increased NO generation. Decreased GS immunoreactivty observed in this study provides a reason for the decreased GS activity in addition to the nitration of GS which was reported earlier. The decreased GS immunoreactivty observed in this study and decreased activity and mRNA expression (reported earlier) may contribute for prolonged availability of glutamate for excitotoxicity.

\section{Acknowledgements}

This study received financial support from Universiti Sains Malaysia-Research University grant (A/C No: 1001/PSKBP/8120196). The parts of the study were presented in $36^{\text {th }}$ Annual Conference of the Malaysian Society for Biochemistry \& Molecular Biology, $27^{\text {th }}-28^{\text {th }}$ July 2011, Selangor, Malaysia, $22^{\text {nd }}$ Malaysian Association of Clinical Biochemists Conference, $2^{\text {nd }}-3^{\text {rd }}$ July 2012, Kula Lumpur, and International Conference on Natural Products 2013, $4^{\text {th }}-6^{\text {th }}$ March 2013, SACC, Selangor, Malaysia.

\section{References}

[1] Zhang, W.Y., Gotoh, T., Oyadomari, S. and Mori, M. (2000) Co Induction of Inducible Nitric Oxide Synthase and Arginine Recycling Enzymes in Cytokine-Stimulated PC 12 Cells and High Output Production of Nitric Oxide. Molecular Brain Research, 83, 1-8. http://dx.doi.org/10.1016/S0169-328X(00)00154-6

[2] Kawahara, K., Gotoh, T., Oyadomari, S., Kajizono, M., Kuniyasu, A., Ohsawa, K., Imai, Y., Kohsaka, S., Nakayama, H. and Mori, M. (2001) Co-Induction of Argininosuccinate Synthetase, Cationic Amino Acid Transporter-2, and Nitric Oxide Synthase in Activated Murine Microglial Cells. Molecular Brain Research, 90, 165-173. http://dx.doi.org/10.1016/S0169-328X(01)00100-0

[3] Sperk, G. (1994) Kainic Acid Seizures in the Rat. Progress in Neurobiology, 42, 1-32. 
http://dx.doi.org/10.1016/0301-0082(94)90019-1

[4] Itoh, K. and Watanabe, M. (2009) Paradoxical Facilitation of Pentylenetetrazole-Induced Convulsion Susceptibility in Mice Lacking Neuronal Nitric Oxide Synthase. Neuroscience, 159, 735-743. http://dx.doi.org/10.1016/j.neuroscience.2008.12.040

[5] Szatkowski, M. and Attwell, D. (1994) Triggering and Execution of Neuronal Death in Brain Ischaemia: Two Phases of Glutamate Release by Different Mechanisms. Trends in Neuroscience, 17, 359-365. http://dx.doi.org/10.1016/0166-2236(94)90040-X

[6] Bidmon, H.J., Gorg, B., Palomero-Gallagher, N., Schleicher, A., Haussinger, D., Speckmann, E.J. and Zilles, K. (2008) Glutamine Synthetase Becomes Nitrated and Its Activity Is Reduced during Repetitive Seizure Activity in the Pentylenterazole Model of Epilepsy. Epilepsia, 49, 1733-1748. http://dx.doi.org/10.1111/j.1528-1167.2008.01642.x

[7] Hammer, J., Alvestad, S., Osen, K.K., Skare, O., Sonnewald, U. and Ottersen, O.P. (2008) Expression of Glutamine Synthetase and Glutamate Dehydrogenase in the Latent Phase and Chronic Phase in Kainite Model of Temporal Lobe Epilepsy. Glia, 56, 856-868. http://dx.doi.org/10.1002/glia.20659

[8] Van Gassen, K.L.I., van der Hel, W.S., Hakvoort, T.B.M., Lamers, W.H. and Graan, P.N.E. (2009) Haploin Sufficiency of Glutamine Synthetase Increases Susceptibility to Experimental Febrile Seizures. Genes Brain and Behavior, 8, 290-295. http://dx.doi.org/10.1111/j.1601-183X.2008.00471.X

[9] Swamy, M., Wan Roslina, W.Y., Sirajudeen, K.N.S., Zulkarnain, M. and Chandran, G. (2011) Decreased Glutamine Synthetase, Increased Citrulline-Nitric Oxide Cycle Activities and Oxidative Stress in Different Regions of Brain in Epilepsy Rat Model. Journal of Physiology and Biochemistry, 67, 105-113. http://dx.doi.org/10.1007/s13105-010-0054-2

[10] Milatovic, D., Gupta, R.C. and Dettbarn, W.D. (2002) Involvement of Nitric Oxide in Kainic Acid-Induced Excitotoxicity in Rat Brain. Brain Research, 957, 330-337. http://dx.doi.org/10.1016/S0006-8993(02)03669-7

[11] Sadasivudu, B. and Lajtha, A. (1970) Metabolism of Amino Acids in Incubated Slices of Mouse Brain. Journal of Neurochemistry, 17, 1299-1311. http://dx.doi.org/10.1111/j.1471-4159.1970.tb03379.x

[12] Koo, C.L., Kok, L.F., Lee, M.Y., Wu, T.S., Cheng, Y.W., Hsu, J.D., Ruan, A., Chao, K.C. and Han, C.P. (2009) Scor-

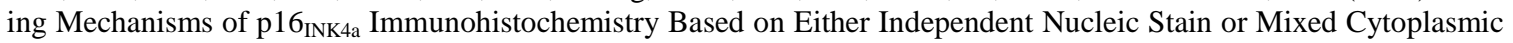
with Nucleic Expression Can Significantly Signal to Distinguish between Endocervical and Endometrial Adenocarcinomas in a Tissue Microarray Study. Journal of Translational Medecine, 7, 25. http://dx.doi.org/10.1186/1479-5876-7-25

[13] Maiti, P., Singh, S.B. and Ilavazhagan, G. (2010) Nitric Oxide System Is Involved in Hypobaric Hypoxia-Induced Oxidative Stress in Rat Brain. Acta Histochemica, 112, 222-232. http://dx.doi.org/10.1016/j.acthis.2008.10.005

[14] Carriedo, S.G., Sensi, S.L., Yin, H.Z. and Weiss, J.H. (1998) Rapid $\mathrm{Ca}^{2+}$ Entry through $\mathrm{Ca}^{2+}$ Permeable AMPa/Kainite Channels Triggers Marked Intracellular $\mathrm{Ca}^{2+}$ Rises and Consequent Oxygen Radical Production. Journal of Neuroscience, 18, 7727-7738.

[15] Guix, F.X., Uribesalgo, M., Coma, M. and Munoz, F.J. (2005) The Physiology and Patophysiology of Nitric Oxide in the Brain. Progress in Neurobiology, 76, 126-152. http://dx.doi.org/10.1016/j.pneurobio.2005.06.001

[16] Swamy, M., Sirajudeen, K.N.S. and Chandran, G. (2009) Nitric Oxide [NO] Citrulline-NO Cycle Enzymes, Glutamins Synthetase and Oxidative Status in Kainic Acid-Mediated Excitotoxicity in Rat Brain. Drug and Chemical Toxicology, 32, 326-331. http://dx.doi.org/10.1080/01480540903130641

[17] Swamy, M., Wan Roslina, W.Y., Intan, N.M.Z., Sirajudeen, K.N.S., Zulkarnain, M. and Chandran, G. (2011) Co-Expression of Citrulline-Nitric Oxide Cycle Enzymes and Decreased Glutamine Synthetase Expression in Different Regions of Brain in Epilepsy Rat Model. African Journal of Pharmacy and Pharmacology, 5, 1522-1529. http://dx.doi.org/10.5897/AJPP11.279

[18] Dong, X.X., Wang, Y. and Qin, Z.H. (2009) Molecular Mechanisms of Excitotoxicity and Their Relevance to Pathogenisis of Neurodegenerative Diseases. Acta Pharmocologica Sinica, 30, 379-387. http://dx.doi.org/10.1038/aps.2009.24

[19] Messripour, M. and Mesripour, A. (2011) Effects of Vitamin B6 on Age Associated Changes of Rat Brain Glutamate Decarboxylase Activity. African Journal of Pharmacy and Pharmacology, 5, 454-456. http://dx.doi.org/10.5897/AJPP11.024

[20] Girard, G., Giguere, J.-F. and Butterworth, R.F. (1993) Region Selective Reductions in Activities of Glutamine Synthetase in Rat Brain Following Portacaval Anastomosis. Metabolic Brain Diseases, 8, 207-215. http://dx.doi.org/10.1007/BF01001062

[21] Rose, C. and Felipo, V. (2005) Limited Capacity for Ammonia Removal by Brain in Chronic Liver Failure: Potential Role of Nitric Oxide. Metabolic Brain Diseases, 20, 275-283. http://dx.doi.org/10.1007/s11011-005-7906-4

[22] Rodrigo, R. and Felipo, V. (2007) Control of Brain Glutamine Synthesis by NMDA Receptors. Frontiers in Bioscience, 
12, 883-890. http://dx.doi.org/10.2741/2110

[23] Kosenko, E., Llansola, M., Montoliu, C., Monfort, P., Rodrigo, R., Hernandez-Viadel, M., Erceg, S., Sanchez-Perez, A.M. and Felipo, V. (2003) Glutamine Synthetase Activity and Glutamine Content in Brain: Modulation by NMDA Receptors and Nitric Oxide. Neurochemistry International, 43, 493-499.

http://dx.doi.org/10.1016/S0197-0186(03)00039-1

\section{List of Abbreviations}

ABC: Avidin-Biotin Complex

AL: Argininosuccinate Lyase

AS: Argininosuccinate Synthetase

AMPA/KA: $\alpha$-Amino-3-Hydroxy-5-Methyl-4-Isoxazolepropionic Acid/Kainic Acid

BS: Brain Stem

CB: Cerebellum

CC: Cerebral Cortex

CNS: Central Nervous System

GS: Glutamine Synthetase

KA: Kainic Acid

NMDA: N-Methyl-D-Aspartate

NOS: Nitric Oxide Synthase

iNOS: Induceble-Nitric Oxide Synthase

nNOS: Neuronal-Nitric Oxide Synthase

PTZ: Pentylenetetrazole 
Scientific Research Publishing (SCIRP) is one of the largest Open Access journal publishers. It is currently publishing more than 200 open access, online, peer-reviewed journals covering a wide range of academic disciplines. SCIRP serves the worldwide academic communities and contributes to the progress and application of science with its publication.

Other selected journals from SCIRP are listed as below. Submit your manuscript to us via either submit@scirp.org or Online Submission Portal.
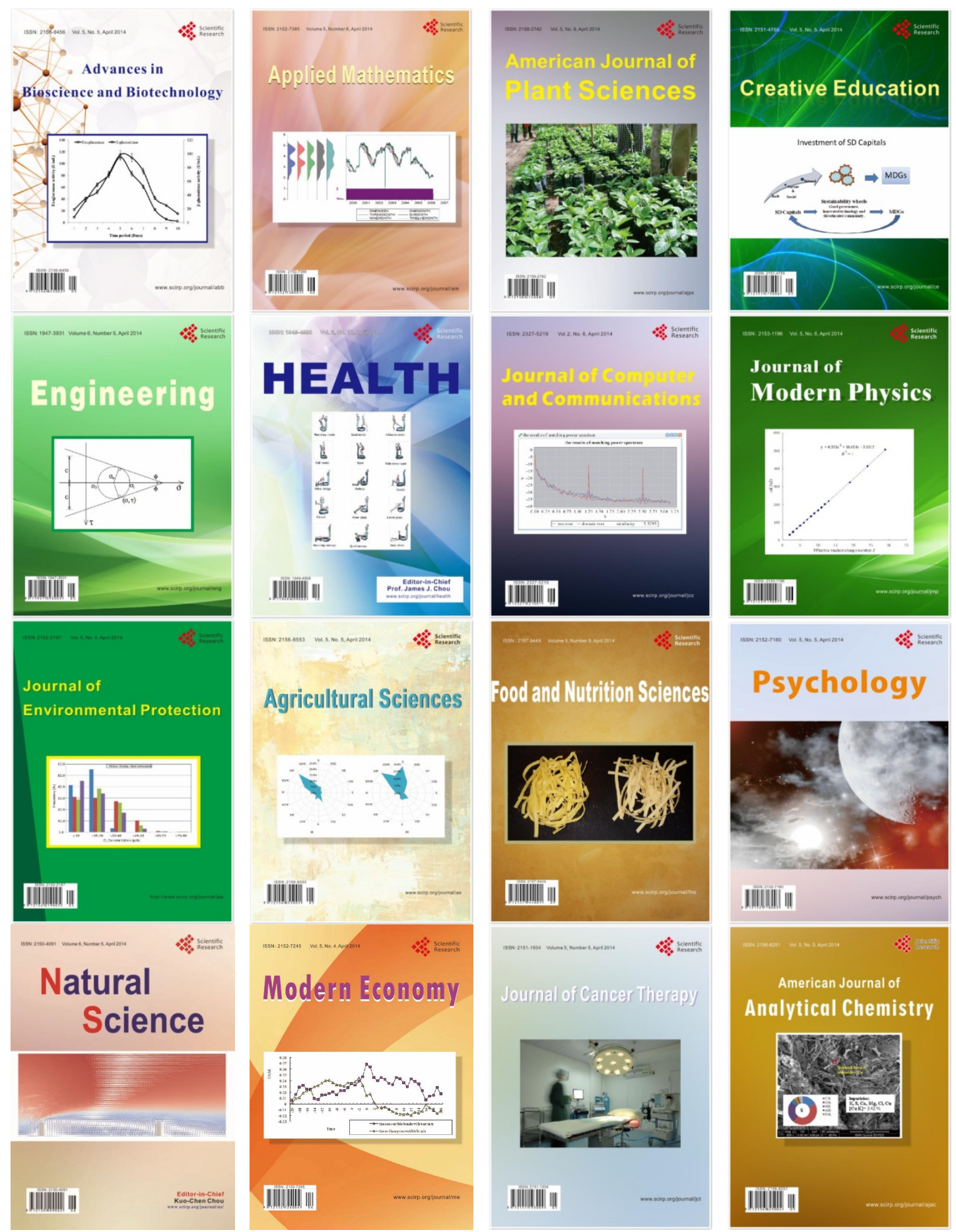